\title{
DESMOID TUMOR AS A DIAGNOSTIC OF GREATER TROCHANTERIC PAIN SYNDROME - CASE REPORT
}

Heloísa Rodrigues Silva Catalá1,^, Andressa Higa Shinzato ${ }^{1}$, Alcânia Walburga de Souza Pereira², Edgard Torres dos Reis Neto ${ }^{1}$, Felipe de Lacerda Veiga ${ }^{1}$, Germana Ribeiro Araujo Carneiro de Lucena ${ }^{1}$, Guilherme Pereira Carlesso ${ }^{1}$, Isadora Medina ${ }^{1}$, Marcela Mingues Castelar Pinheiro ${ }^{1}$, Pedro Matos $^{1}$, Stella Falcadi Vendramine ${ }^{1}$

1. Universidade Federal de São Paulo, São Paulo (SP), Brazil. 2. Hospitical Samaritano Américas Serviços Médicos, São Paulo (SP), Brazil.

*Corresponding author: catalaheloisa@gmail.com

\section{BACKGROUND}

Trochanteric pain can affect individuals of all ages and is reported in $10-25 \%$ of the world's population. The differential diagnosis is broad, including both intra- and extra-articular diseases, such as iliotibial band thickening, bursitis, and tendinopathy of gluteus medius and minimus muscles. We report a case of a young woman with complain of pain in the region of greater trochanteric without satisfactory response to infiltration of local corticosteroids and physiotherapy who was diagnosed with an iliotibial desmoid tumor (DT).

\section{CASE REPORT}

A 33-year-old Afrodescendant female, with no previous personal or family comorbidities and no history of continuous medication use, who presented mechanical insidious pain in the right trochanteric region for six months, with no associated trauma and only partial improvement with analgesics. On physical examination, she presented pain on palpation of the greater trochanter without palpable tumor or local inflammatory signs. Considering the hypothesis of trochanteric painful syndrome, local infiltration with betamethasone and xylocaine was performed and physiotherapy was prescribed without clinical improvement. Magnetic resonance imaging was requested, which demonstrated an expansive lesion with its epicenter in the proximal portion of the iliotibial tract, with a heterogeneous signal and contrast enhancement, extending to the muscular gluteal and establishing contact with the external bone surface of the iliac wing, measuring $7.2 \times 4.4 \times 3.8 \mathrm{~cm}$ suggesting the possibility of a DT (Fig. 1). Biopsy of the lesion confirmed the diagnosis of DT. Treatment with nonsteroid anti-inflammatory drugs (NSAIDs) for 3 months was initiated for subsequent evaluation of local surgical treatment.
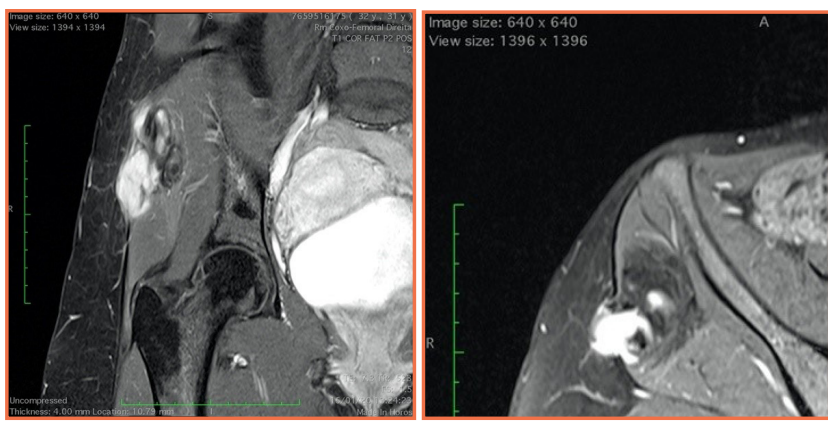

Figure 1. Expansive lesion with epicenter in the proximal portion of the iliotibial tract, with heterogeneous sign and contrast enhancement.

\section{CONCLUSION}

Desmoid tumor is a rare disease characterized by a locally aggressive monoclonal, fibroblastic proliferation leading to a variable and often unpredictable clinical course. The incidence is estimated to be 5-6 cases per 1 million of the population per annum with a peak age of 30-40 years and a female predominance. Although a DT is not life-threatening in the vast majority of cases, patients often suffer from pain, functional deficits, psychological problems and a general decrease in quality of life. Due to high rate of recurrence after surgery, there is no consensus about the gold standard treatment which can includes NSAIDs, chemotherapy, radiotherapy and surgery resection. In cases of trochanteric painful syndrome with warning signs or refractory to the initial treatment, imaging should be requested for the differential diagnosis with other etiologies. 
